\title{
Salt body inversion using an optimal transport of the preconditioned matching filter
}

\author{
Bingbing Sun and Tariq Alkhalifah \\ bingbing.sun@kaust.edu.sa, tariq.alkhalifah@kaust.edu.sa \\ Physical Sciences and Engineering \\ King Abdullah University of Science and Technology \\ Thuwal, 23955, Saudi Arabia
}

March 3, 2020

\section{Summary}

The conventional least squares L2 norm is susceptible to local minima if the low wavenumber components of the initial model are not accurate, and this happens more often with data corresponding to salt bodies. Deconvolution of the predicted and observed data offers an extended space comparison, which is more global. The matching filter calculated from the deconvolution has energy focussed at zero-lag, like a Dirac delta function, when the velocity model is accurate, and the predicted data matches the observed one. We utilize a framework for designing a misfit function by measuring the Wasserstein distance W2 between the resulting matching filter and a representation of the Dirac delta function based on the optimal transport theory. Unlike data, the matching filter can be easily transformed into a distribution satisfying the requirement of the optimal transport theory. This optimal transport between these two distributions leads to minimizing the least squares difference of the mean and variance of the distributions. If the objective for the matching filter is a Dirac delta function, i.e., with both zero mean and variance, the optimization reduces to the adaptive waveform inversion (AWI) misfit. Along with a total variation constraint, we show that this method can 


\section{Introduction}

Designing a robust misfit function which can mitigate cycle skipping in FullWaveform Inversion (FWI) is highly important. The conventional $l_{2}$ norm misfit function though simple, and potentially high resolution, is a local comparison of the data and prone to cycle skipping. Thus, many have addressed the cycleskipping problem by introducing more robust misfit functions, which try to compare the data in a more global way such as a matching filter based misfit function [Luo and Sava, 2011, Warner and Guasch, 2016, Sun and Alkhalifah, 2018b] or a optimal transport misfit function [Engquist and Froese, 2014, Métivier et al., 2016, Yang et al., 2018, Sun and Alkhalifah, 2018a, Sun and Alkhalifah, 2019].

In the matching filter approach, a matching filter is computed first by deconvoving of the predicted by the measured data. If the velocity model is accurate, the non-zero coefficients of the filter would focus at the zero lag, thus a misfit function can be designed by penalizing the coefficients of the filter at none-zero lag to update the model. Current applications of the optimal transport transform the measured and predicted data to distributions first and then formulate the misfit function by Wasserstein distance between those two distributions. One critical difficulty in applying the optimal transport is preconditioning the seismic data to formulate a distribution, i.e., the resulting two distributions should both be nonnegative and have equal mass. Several precondition methods such as the affine-scale or the exponential coding has been proposed, but since these methods modify the data directly, they tend to change the amplitude or phase of the seismic data. More importantly, the convexity feature that the Wasserstein distance has with respect to time shifts between two signals is lost due to such improper precondition of the seismic data.

In this abstract, we combine the matching filter and the optimal transport theory to design a robust misfit function for FWI. Unlike the conventional matching filter approachs which use a penalty to construct the misfit function, we use optimal transport theory to measure the focusing of the resulting matching filter by measuring the Wasserstein $W_{2}$ distance between a preconditioned matching filter and a representation of the Dirac delta function. The new misfit function shows multiple benefits: 1) It is a global comparison method based on a matching filter unlike the $l_{2}$ norm which is a local measure and thus, it can help in mitigating cycle skipping. 2) It uses the convex Wasserstein distance rather than a simple penalty to measure the focusing property of the resulting matching filter. 3) The resulting matching filter can be easily preconditioned to form a distribution without issues, i.e., using squareness to make it positive and division by its sum to make the mass equals to 1 . Specially, we precondition the resulting matching filter, not the original seismic data. As the most valuable information in the matching filter is how its coefficients distributed over the time lags, the preconditioning of the matching filter will not change this. 4) As we show later, adaptive waveform inversion (AWI) can be considered as a special case of our proposed misfit function which tries to minimize the sum of

the mean and variance of the resulting matching filter distribution. Using total variation constraint that we relax slowly over iterations, we apply this approach 
to the complex Salt body BP model.

\section{Wasserstein distance between modeled and mea- sured data}

Considering the modeled data $p(t)$ and the measured data $d(t)$, both of which are modified and preconditioned to be distributions. Optimal transport suggests that we can use the Wasserstein $W_{2}$ distance to design a misfit function [Yang et al., 2018]:

$$
W_{2}^{2}(p, d)=\min _{T} \int|t-T(t)|^{2} p(t) d t,
$$

where $T$ is the transport plan, which maps the mass in distribution $p$ into $d$, i.e., $\int T\left(t, t^{\prime}\right) d t^{\prime}=d(t), \quad \int T\left(t, t^{\prime}\right) d t=p\left(t^{\prime}\right)$. For the 1D problem, i.e., the optimal transport is applied per trace, an explicit formula exists for the Wasserstein $W_{2}$ distance [Villani, 2003], in which the corresponding optimal transport (OT) misfit function is given by:

$$
J_{\mathrm{OT}}=W_{2}^{2}(p, d)=\int\left|t-D^{-1}(P(t))\right|^{2} p(t) d t,
$$

where $D^{-1}$ is the inverse function corresponding to $D$, and $D$ and $P$ are the cumulative distribution functions for the measured and modeled data, respectively: $D(t)=\int_{0}^{t} d\left(t^{\prime}\right) d t^{\prime}, \quad P(t)=\int_{0}^{t} p\left(t^{\prime}\right) d t^{\prime}$.

\section{Wasserstein distance between matching filter and Dirac delta function}

In this section, we propose to compute a matching filter by deconvolving the measured data from the modeled one first. After a proper precondition of the resulting matching filter to fulfill the requirements for a distribution, we measure the Wasserstein $W_{2}$ distance between the resulting matching filter and a target given by, e.g., a Dirac delta function, which is a distribution. In this approach, we avoid the drawbacks involved in modifying the original seismic data directly to meet the requirements for a distribution.

Given measured data $d(t)$ and modeled data $p(t)$, the matching filter $w(t)$ satisfies:

$$
d(t) * w(t)=p(t)
$$

where $*$ denotes the convolution operation. [Luo and Sava, 2011] proposed a matching filter (MF) based misfit function that penalizes energy beyond the zero lag of the resulting matching filter $w(t)$ :

$$
J_{\mathrm{MF}}=\int A^{2}(t) w^{2}(t) \mathrm{d} t
$$


where $A(t)$ is a penalty function, e.g., $A(t)=|t|$. The misfit function of equation 4 has a potential drawback: the misfit function is sensitive to the amplitude of the resulting matching filter. We can reduce the misfit value by decreasing the amplitude of the matching filter $w(t)$. However, the expected evolution of the matching should reduce its amplitude at large time lag while increasing its amplitude at small time lags and gradually focus the energy to zero time lag. However the misfit function of equation 4 will not drive the energy to zero lag, rather it might penalize the value at all lags to be zero and potentially lead to no reflections in the modeled data. To resolve this limitation, we use the Wasserstein $W_{2}$ norm to measure the distance between the matching filter $w(t)$ and the Dirac delta function. To fulfill the requirements of the optimal transport theory, we need to transform $w(t)$ to a distribution. We elect, here, to square it and normalize it as follows:

$$
\tilde{w}(t)=\frac{w^{2}(t)}{\int_{0}^{1} w^{2}(t) d t}=\frac{w^{2}}{\|w\|_{2}^{2}} .
$$

Other preconditioning, like the envelope, may also be used. The transformation of the matching filter to a probability distribution is crucial: Firstly, it is a strict requirement for application of the optimal transport theory. Secondly, it would make the resulting misfit function insensitive to the amplitude of the matching filter, and thus, avoids the problem faced by the conventional penalty-based matching filter misfit function of equation 4.

When the model parameters are accurate, the resulting matching filter reduces to a Dirac delta function. This implies that the Dirac delta function $\delta(t)$ could be the target. Based on the theory of optimal transport, we use equation 2 directly, and the resulting optimal transport based matching filter (OTMF) misfit function is formulated as:

$$
J_{\mathrm{OTMF}}=W_{2}^{2}(\tilde{w}, \delta)=\frac{\int\left|t-\Delta^{-1}(\tilde{W}(t))\right|^{2} w^{2}(t) d t}{\|w\|_{2}^{2}} .
$$

Here, we use $\Delta$ and $\tilde{W}(t)$ to denote the cumulative distribution functions for the Dirac delta function $\delta(t)$ and the modified matching filter $\tilde{w}(t)$, respectively: $\Delta=$ $\int_{0}^{t} \delta\left(t^{\prime}\right) d t^{\prime}, \quad \tilde{W}(t)=\int_{0}^{t} \tilde{w}\left(t^{\prime}\right) d t^{\prime}$. Here, $\Delta^{-1}$ is the inverse function for the cumulative distribution function $\Delta$. Because of the singularity involved in the Dirac delta function, in practice, we use a Gaussian function with a small standard deviation, which is a practical form of an approximated functional representation of the Dirac delta function. 


\section{Relationship with Adaptive Waveform Inver- sion (AWI)}

[Warner and Guasch, 2016] proposed to add a normalization term in the conventional penalty-based misfit of equation 4 and proposed the AWI misfit:

$$
J_{\mathrm{AWI}}=\frac{\int t^{2} w^{2}(t) \mathrm{d} t}{\|w\|_{2}^{2}}=\int t^{2} \tilde{w} \mathrm{~d} t=\mathrm{E}\left(X^{2}\right)
$$

here, we select penalty $A(t)=|t|$ in equation 4 and we use the preconditioned matching filter $\tilde{w}$ notation of equation 5 . In statistics, the term $\int t^{2} \tilde{w} \mathrm{~d} t$ is the second moment of the distribution $\tilde{w}$, i.e., $\mathrm{E}\left[X^{2}\right]$ where $X$ denotes the random variable with probability distribution $\tilde{w}$.

We use $Y$ to denote the random variable corresponding to the Dirac delta function. Both of the matching filter distribution and Dirac delta function can be approximated by Gaussian distributions, i.e., $X \sim \mathcal{N}(\mu, \sigma)$ and $Y \sim$ $\mathcal{N}\left(\mu_{0}, \sigma_{0}\right)$. Here $\mu=\mathrm{E}[X]$ and $\mu_{0}=\mathrm{E}[Y]$ denote the mean value while $\sigma^{2}=$ $\mathrm{E}\left[(X-\mu)^{2}\right]$ and $\sigma_{0}^{2}=\mathrm{E}\left[\left(Y-\mu_{0}\right)^{2}\right]$ are the variance of the corresponding distributions. Based on the proposed framework, if we consider the Wasserstein $W_{2}$ distance between those two Gaussian distributions, we immediately obtain [Olkin and Pukelsheim, 1982]:

$$
W_{2}^{2}\left[(\mu, \sigma),\left(\mu_{0}, \sigma_{0}\right)\right]=\left(\mu-\mu_{0}\right)^{2}+\left(\sigma-\sigma_{0}\right)^{2}
$$

Dirac delta function can be considered as a Gaussian function with zero mean and variance, i.e., $Y \sim \mathcal{N}(0,0)$, and by setting both $u_{0}$ and $\sigma_{0}$ in equation 8 to zero, we obtain:

$$
W_{2}^{2}[(\mu, \sigma),(0,0)]=\mu^{2}+\sigma^{2}=\mathrm{E}[X]+\mathrm{E}\left[(X-\mu)^{2}\right]=\mathrm{E}\left[X^{2}\right]
$$

We can observe that the resulting formula gives the same form of $\mathrm{E}\left[X^{2}\right]$ as the AWI misfit function of equation 7 . This is due to $\mathrm{E}[X]+\mathrm{E}\left[(X-\mu)^{2}\right]=$ $\mathrm{E}\left[X^{2}\right]$ for Gaussian distributions. As a result, we can say that AWI is actually trying to minimize the sum of the square of the mean and variance of the resulting matching filter distribution. The normalization term introduced in AWI is trying to fulfill the requirement for a probability distribution according to our analysis.

\section{Results}

Using our proposed approach given by equation 6 , we invert for a complex BP salt body model. The true velocity shown in Figure 1a extends $4.8 \mathrm{~km}$ in depth and $21.6 \mathrm{~km}$ laterally. We choose an initial model free of the salt body as shown in Figure 1b. The source wavelet is a Ricker wavelet with a $7 \mathrm{~Hz}$ peak frequency and bandpass filtered between $2 \mathrm{~Hz}$ and $10 \mathrm{~Hz}$. We invert the dataset directly in the time domain without using low to high frequency continuation. Applying 


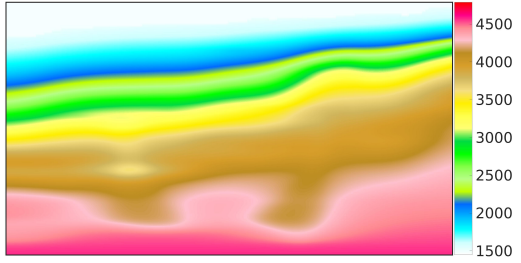

(a)

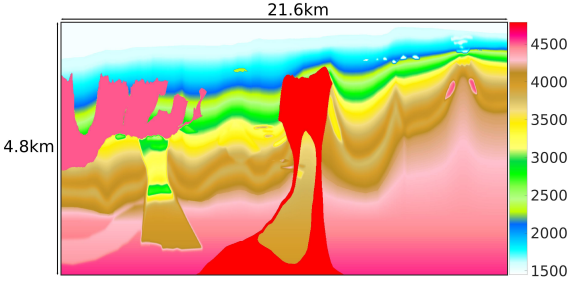

(b)

Figure 1: a) The true velocity; b) The initial velocity for the modified BP model.

FWI to models that contain salt bodies is challenging. Here, we include a total variation (TV) regularization to help with producing a blocky model to fit the data. The TV regularization is set relatively strong at the begining and is gradually reduced over iterations. Figure 2a shows the inversion result using the conventional $l_{2}$ norm, and it is severally cycle-skipped in such a frequency band. Figure $2 \mathrm{~b}$ shows the inverted model using the proposed method, and due to low illumination at the boundary, there are some artifacts, however, the shape of the salt is well resolved. The inverted subsalt area also shows high accuracy.

\section{Conclusions}

We proposed a misfit function, that is based on the optimal transport between a matching filter, corresponding to fitting the observed to the predicted data, and a distribution in which energy is focused at zero lag, like the Dirac delta function. The matching filter, unlike data, is better suited to form a distribution, which is required for the optimal transport theory. Based on this framework, the misfit function of AWI can be interpreted as a minimization of the sum of squares of the mean and variance of the resulting matching filter distribution. The critical normalization term in AWI is a nature requirement for a distribution in optimal transport theory. Salt body inversion of the BP model verified the effectiveness of the proposed misfit function in mitigating the cycle-skipping problem.

\section{Acknowledgements}

We thank KAUST for supporting this research and members of SWAG for useful discussions.

\section{References}

[Engquist and Froese, 2014] Engquist, B. and Froese, B. [2014] Application of the Wasserstein metric to seismic signals. Communications in Mathematical 


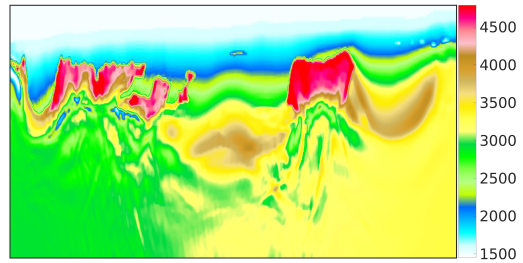

(a)

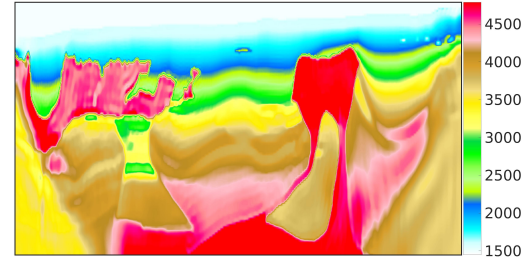

(b)

Figure 2: The inverted velocity using a) the $l_{2}$ norm misfit function; the proposed optimal transport matching-filter misfit function for the modified BP model.

Sciences, 9(1), no. 1, 79-88.

[Luo and Sava, 2011] Luo, S. and Sava, P. [2011] A deconvolution based objective function for wave equation inversion. SEG Technical Program Expanded Abstracts, 2788-2792.

[Métivier et al., 2016] Métivier, L., Brossier, R., Mérigot, Q., Oudet, E. and Virieux, J. [2016] Measuring the misfit between seismograms using an optimal transport distance: application to full waveform inversion. Geophysical Journal International, 205(1), no. 1, 345-377.

[Olkin and Pukelsheim, 1982] Olkin, I. and Pukelsheim, F. [1982] The distance between two random vectors with given dispersion matrices. Linear Algebra and its Applications, 48, $257-263$.

[Sun and Alkhalifah, 2018a] Sun, B. and Alkhalifah, T. [2018a] The application of an optimal transport to a preconditioned data matching function for robust waveform inversion. SEG Technical Program Expanded Abstracts.

[Sun and Alkhalifah, 2018b] Sun, B. and Alkhalifah, T. [2018b] Mitigate Cycle Skipping In FWI: A Generalized Instanatenous Travel-Time Approach. 80th EAGE Conference and Exhibition.

[Sun and Alkhalifah, 2019] Sun, B. and Alkhalifah, T. [2019] A robust waveform inversion using a global comparison of modeled and observed data. The Leading Edge, accepted.

[Villani, 2003] Villani, C. [2003] Topics in optimal transportation, Graduate studies in math- ematics:. American Mathematical Society, 58, 1-145.

[Warner and Guasch, 2016] Warner, M. and Guasch, L. [2016] Adaptive waveform inversion: Theory. Geophysics, 81(6), no. 6, R429-R445.

[Yang et al., 2018] Yang, Y., Engquist, B., Sun, J. and Hamfeldt, B.F. [2018] Application of optimal transport and the quadratic Wasserstein metric to full-waveform inversion. Geophysics, 83(1), no. 1, R43-R62. 